sets of observations led Laver and Webster to the conclusion that the Hong Kong virus might have arisen by recombination between an existing human virus and an animal or avian virus; the first donating neuraminidase subunits, the second donating haemagglutinin subunits to the recombinant Hong Kong virus.

In 1968 and 1969 it had also been shown that two strains of influenza virus that in 1963 had been isolated from ducks and horses were serologically related to the human Hong Kong virus and that this relationship was probably mediated only through the haemagglutinin subunits of the three viruses, avian, equine and human, because the neuraminidase subunits of the three proved to be quite unrelated.

What Laver and Webster have now shown is that the duck, equine and Hong Kong influenza viruses do indeed have haemagglutinin subunits that are serologically cross-reacting. Furthermore, by comparing peptide maps of the haemagglutinin subunits of the three viruses they have established that the light polypeptide chains of the subunits are virtually identical whereas the heavy chains differ significantly. It seems, therefore, that the Hong Kong virus might well have arisen by recombination between a human virus and an equine (or avian) virus related to equine 2 (or duck/Ukraine) influenza virus, and Webster and his colleagues have apparently obtained such human/ animal (or avian) recombinants in experiments which mimic natural conditions of transmission.

Downie and Laver (ibid., 259) also believe that there are probably plenty of as yet undetected avian influenza viruses in existence from which human pandemic strains may arise at any time as a result of such recombination. Indeed, they have isolated such a virus from an apparently healthy Australian shearwater. This avian virus is a type A virus, and of course pandemics of type A viruses occur in man. The haemagglutinin subunit of this shearwater virus belongs to a known avian type, but the neuraminidase subunit of the virus is antigenically distinct from all other known avian influenza viruses.

This virus is only the second type A influenza virus ever to have been isolated from a wild bird ; it did not apparently cause any disease in its host and it has a novel neuraminidase subunit. Presumably many wild birds harbour infections by benign influenza viruses and they probably provide a pool of viruses which by recombination with existing human viruses can give rise at any time to new virulent strains able to cause pandemics in man. In short, epidemics of influenza seem certain to continue to plague man for the foreseeable future.
ANIMAL BEHAVIOUR

\section{Aggressive Anemones}

from our Animal Behaviour Correspondent

AgGressiveness is not a trait very obviously associated with sea anemones. Yet Francis (Biol. Bull., 144, 73 ; 1973) has not only found overt aggression in the Californian anemone Anthopleura elegantissima, but also produces evidence that they are selectively aggressive to anemones of a different genotype from themselves.

A most striking feature of the spatial distribution of the anemones is that they live in clumps: there are definite patches of animals interspersed with anemonefree spaces. A given clump is made up of individuals all of the same colour pattern and of the same sex. The anemones are known to reproduce asexually by longitudinal fission, so that the different clumps are almost certainly groups of genetically identical individuals (clones). When taken into the laboratory and mixed together, the anemones segregate back into their clones.

Contact between genetically different individuals was found to lead to an elaborate series of aggressive behaviours, one or both animals being damaged in the process. First, the tentacles touch and withdraw repeatedly. If the anemones are from the same clone, the animals move toward each other, their tentacles interlacing. But if they are from different clones, this touching and withdrawing of tentacles is followed by distension of the acrorhagi — rounded objects situated beneath the outer layer of the tentacles. The inflated acrorhagi (now cone-shaped) are drawn upwards and then swept downwards towards the victim and wiped against its body. Tissue touched by the acrorhagi is damaged by the stinging cells they contain and begins to deteriorate. The victim either moves away or, if it is unable to do so, is killed.

Anthopleura shows this behaviour neither to species upon which it preys (for example, small mussels) nor to its predators (for example, sea slugs) and the curious clumped distribution of these anemones over the rocks would seem to be attributable to their own aggressive behaviour which has the effect of spacing them out from other anemones of alien genotype.

\section{PLASMAS}

\section{Latest with Lasers} A CONFERENCE on laser produced plasmas, organized jointly by the Quantum Electronics and Plasma Physics Groups of the Institute of Physics, was held from April 17 to 18 at the University of Hull. The keen interest shown in the subject fully reflects the rapid increase in international activity since last year's publication of some

\title{
Shutoff Mechanism after Phage Infection
}

SHUTOFF of host transcription in Escherichia coli cells after phage T4 infection is prevented by chloramphenicol and it has been assumed therefore that a T4 protein(s) synthesized after infection is responsible for shutoff. But attempts to find the presumptive gene(s) which code(s) for such T4 protein(s) have been unsuccessful. The problem could be solved by study of a mutant defective in host shutoff. An RNA polymerase mutant (Rif ${ }^{\mathrm{R}}$-2) has been found which makes host RNA as late as 6-8 min after infection, and in next Wednesday's Nature New Biology (May 30), Snyder describes experiments with this mutant which cast a new light on the possible mechanism of host shutoff in phage infected cells.

Snyder investigated differences in sedimentation rate and efficiency of utilization of various templates in the RNA polymerase of infected and uninfected cells. These differences appear very early in wild type infection, but are delayed after infection of mutants in which shutoff of host transcription is also delayed. The effect is independent of chloramphenicol treatment.

The RNA polymerase from uninfected cells sediments as a broad peak in glycerol gradients. Different parts of the polymerase peak transcribe different templates with varying efficiency, showing that the polymerase peak is heterogeneous. In particular, a rapidly sedimenting polymerase activity disappears quickly after T4 infection and the polymerase peak becomes more homogeneous. In the Rif $^{\mathrm{R}}-2$ mutant, however, the change in the profile of polymerase activity is delayed and even 8 min after phage infection the rapidly sedimenting activity can still be detected. This difference between the polymerase activity after infection of the mutant and wild type cells is independent of chloramphenicol treatment.

These experiments were carried out on crude preparations of RNA polymerase. Snyder suggests that the rapidly sedimenting polymerase may result from weakly bound factors which would be lost during the usual purification procedures and thus have not been detected previously. Thus he proposes that host shutoff is caused by inactivation of these factors which are required for transcription of host DNA in vivo but not in vitro. 